\title{
Enhancement of Spontaneous Emission from CdSe Quantum Dots by 3-D Silica Photonic Crystal
}

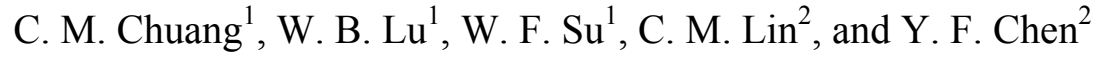 \\ 1. Department of Materials Science and Engineering, National Taiwan University, Taipei, 106, Taiwan \\ 2. Department of Physics, National Taiwan University, Taipei, 106, Taiwan
}

\begin{abstract}
We have demonstrated that the luminescence properties of cdse nanoparticles can be manipulated by selfassembled silica photonic crystal. When the emitting wavelength of nanoparticles matches the stop band of photonic crystal, the photoluminescence of the cdse nanoparticles can be greatly enhanced by up to 5 times. by changing the collection angle of photoluminescence measurements, the photoluminescence intensity of cdse embedded in photonic crystal can also be controlled.

Index Terms - Nanotechnology, Photoluminescence, Semiconductor materials.
\end{abstract}

\section{INTRODUCTION}

Yablonovitch [1] and John [2] are the first to suggest the concept of photonic crystals in 1987. Photonic crystals are macroscopic dielectric media arranged in periodic structures. They exhibit photonic band gap that is similar to the electronic band gap of the material. We therefore can control the propagation of the light, such as allow it only in certain direction at certain frequencies, or localize light in specific areas. A tremendous application in optical communication is expected. In 1989, Yablonovitch and Gmitter [3] proved the concept experimentally. Since then great attention has been paid to the fabrications and the studies of artificial three-dimensional periodic dielectric structures. Many methods have been developed to fabricate the photonic crystal that include multi-layer lithography [4], multi-beam holographic lithography [5], and self-assembly [6]-[7], etc. Among them, self-assembly is the simplest method to fabricate 3-D photonic crystals. Essentially, monodisperse colloidal spheres can be spontaneously assembled into periodic arrays under certain circumstances. Blanco et al. [8] and Miller et al. [9] have gone a future step to obtain a complete band gap. They have used nanoparticles embedded photonic crystal to improve the refractive index contrast between periodic structures. For the application of photonic crystal, silica capillaries had been stacked, fused together, and drawn down to photonic crystal fiber [10], and then it have been possible to greatly widen the transmission bands by fabricating a different structure, a Kagome lattice [11].
Colombelli et al. [12] combined photonic and electronic band structure engineering to create a surface-emitting quantum cascade microcavity laser. Noda et al. [13] have made a photonic nanostructure device which light propagating through the waveguide was trapped by the point defect and emitted to free space.

\section{EXPERIMENTAL}

The CdSe nanoparticles were grown by modifying Peng's [14] method. CdO 0.06 g, trioctylphosphine oxide (TOPO) $5 \mathrm{~g}$, and tetradecylphosphonic acid (TDPA) 0.26 $\mathrm{g}$ were loaded in a three neck flask. At about $300{ }^{\circ} \mathrm{C}$, reddish $\mathrm{CdO}$ powder was dissolved into colorless homogeneous solution. By cooling down to $270{ }^{\circ} \mathrm{C}$, then an injection of $0.9 \mathrm{ml} \mathrm{Se}$ solution ( $1.987 \mathrm{~g}$ Se dissolved in $50 \mathrm{ml}$ tributylphosphine (TBP)), high-quality CdSe nanoparticles were obtained. The size of nanoparticles was evaluated by transmission electron microscopy (TEM) to be $4.6 \mathrm{~nm} \pm 10 \%$. The UV-VIS and PL spectra of CdSe show sharp absorption peaks that indicate the nanoparticles close to monodisperse.

Amorphous monodispersive spherical silica particles were synthesized by using sol-gel method [15]. A $20 \mathrm{ml}$ of tetra-ethyl orthosilicate (TEOS) was dissolved in 200 $\mathrm{ml}$ ethanol $(95 \%)$, the solution was held and stirred with a mechanical stirrer in water bath at $30{ }^{\circ} \mathrm{C}$ for $30 \mathrm{~min}$. Then $20 \mathrm{ml}$ ammonia solution (ammonium hydroxide, 28 30 wt $\%$ solution of $\mathrm{NH}_{3}$ in water) was added into the TEOS solution, and stirred for 2 hours. After 2 hours of the reaction, monodispersive silica colloid solution was obtained. Then the silica colloid solution was centrifuged at $5000 \mathrm{rpm}$ for $10 \mathrm{~min}$ to separate the silica particles from liquid. The powder was then washed by $200 \mathrm{ml}$ ethanol accompanied with ultrasonication. The washing procedure was repeated three times to ensure the complete removal of reactants. Then the silica powder was dried at $100{ }^{\circ} \mathrm{C}$ for 12 hours.

The silica photonic crystal was prepared using convective method. ${ }^{7}$ Silica powder was redispersed in ethanol with a concentration of $1 \mathrm{wt} \%$ solution. And then 
the glass substrate was immersed into the silica colloid solution vertically. The solution was placed in a $80{ }^{\circ} \mathrm{C}$ oven for 12 hours. After the evaporation of ethanol, the silica photonic crystal was self-assembled onto the vertical substrate.

\section{RESULT AND DISCUSSION}

Fig. 1 shows the scanning electron microscopy (SEM) micrographs of silica photonic crystal infiltrated with CdSe nanoparticles. The silica photonic crystal reveals a hexagonal-type close packing. The CdSe nanoparticles are uniformly distributed inside the silica photonic crystal.

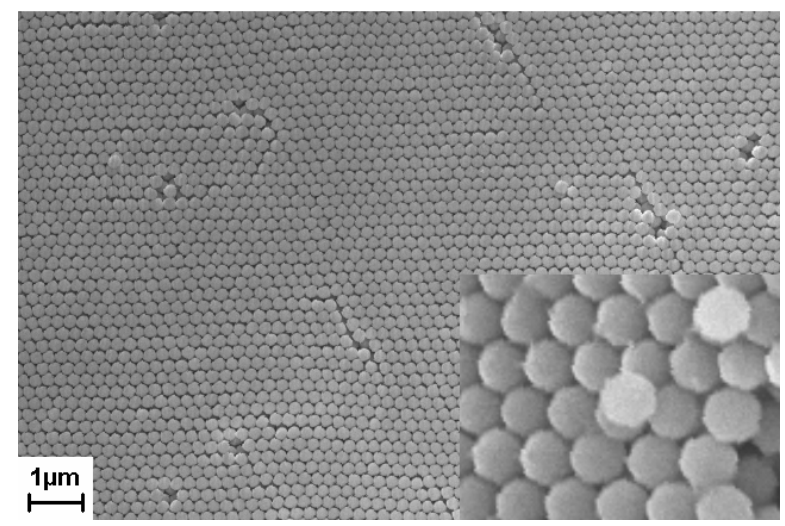

Fig. 1. SEM micrograph of silica photonic crystal infiltrated with $\mathrm{CdSe}$ nanoparticles. The inset shows the CdSe nanoparticles are uniformly distributed inside the photonic crystal.

Colloidal crystals formed from monodisperse spheres exhibit a photonic crystal bandgap. The photonic stop band demonstrates themselves as dips in transmission spectra or as peaks in the corresponding reflection spectra. The spectra position of the stop band depends on (1) the diameter of spheres that built photonic crystal, (2) the angle between the crystal orientation of the sample and the direction of the incident light, and (3) the refractive index constant of the photonic crystal. In this work, optical transmission spectra were measured by UV-VIS spectrometer. Fig. 2 shows transmission spectra of silica photonic crystals at normal incident of the light. As expected from calculation [16], for the f.c.c. lattice, the stop band $\lambda_{\max }$ changes with the spheres diameter D as shown in the following

$$
\lambda_{\max }=2(2 / 3)^{1 / 2} D\left(n^{2}-\sin ^{2} \theta\right)^{1 / 2}
$$

where $\mathrm{n}$ is the refractive index of the assembly particles, $\theta$ is the angle between the incident light and the normal to the substrate. The absorption peaks are changed with the diameters of silica spheres varied from $407-738 \mathrm{~nm}$ as expected.

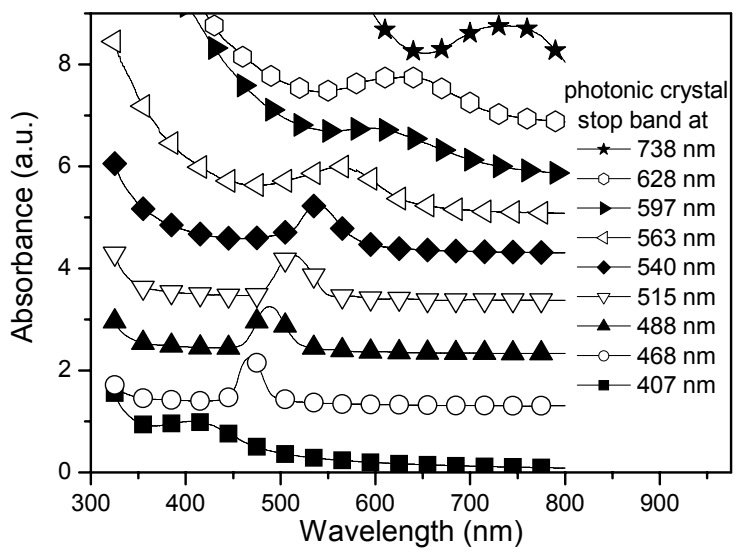

Fig. 2. Transmission spectra of nine samples of silica photonic crystals at normal incident of light. The absorption peaks are changed with the diameters of silica spheres that can be varied from $407-738 \mathrm{~nm}$

Photoluminescence measurements were taken at room temperature under $325 \mathrm{~nm} \mathrm{He}-\mathrm{Cd}$ laser line excitation. There is no luminescence from silica photonic crystal, thus any luminescence observed arises from the CdSe nanoparticles. By selecting the right sphere diameter that matches as the wavelength of the emitting spectrum of CdSe nanoparticles, the photoluminescence of $\mathrm{CdSe}$ nanoparticles can be manipulated by 3-D photonic crystal.

Samples with three different arrangements of CdSe nanoparticles were used to study the manipulation of their photoluminescence by 3D photonic crystals. The sample of the first arrangement was prepared by placing the CdSe solution onto the glass slide, and then evaporated off solvent to obtain thin layer nanoparticles on the glass. The photoluminescence was measured at the same side of CdSe nanoparticles layer. The sample of the second arrangement was prepared by placing the CdSe solution onto the silica photonic crystal, and the nanoparticles were infiltrated into photonic crystal. Then the solvent was evaporated off to obtain the sample. The photoluminescence was measured at the same side of CdSe infiltrated. The sample of the third arrangement was prepared by placing the CdSe nanoparticles onto the glass substrate and the photonic crystal was on the other side of the glass substrate. The photoluminescence measured at the same side of CdSe. The CdSe solution was mixed with $3 \mathrm{~nm}$ and $4.6 \mathrm{~nm}$ nanoparticles and exhibited photoluminescence peak at $517 \mathrm{~nm}$ and $580 \mathrm{~nm}$, respectively. For a photonic crystal assembled from 234 $\mathrm{nm}$ silica spheres, the stop band of photonic crystal for 
normal incident is at about $490 \mathrm{~nm}$. If the photoluminescent peak of $\mathrm{CdSe}$ is matched with transmission peak of photonic crystal then a high coupling between the light emission of CdSe and the stop band of silica photonic crystal will be revealed.

The photoluminescence measurement of CdSe nanoparticles with the first arrangement was used as the blank of experiment. The CdSe is photoluminescent material without silica photonic crystal. The photoluminescence peak at $580 \mathrm{~nm}$ which does not affect by the stopping band of silica photonic crystal, was used as an internal standard to normalize the peak intensity at $517 \mathrm{~nm}$. To eliminate the effect of photonic crystal band on the photoluminescence of the CdSe at $580 \mathrm{~nm}$, we selected the stopping band of photonic crystal band at $490 \mathrm{~nm}$, not at the $517 \mathrm{~nm}$. The photoluminescence intensity at $517 \mathrm{~nm}$ from the second arrangement, and the third arrangement were greatly enhanced by 4 to 5 times to that of the first arrangement as shown in Fig. 3. In the second arrangement, the CdSe nanoparticles covered the silica photonic crystal completely. When the CdSe nanoparticles on the surface of photonic crystal were excited by laser, it emitted the photoluminescence. The collected photoluminescence now includes the emitted radiation reflected by silica photonic crystal because the emission of $\mathrm{CdSe}$ nanoparticles is not allowed to travel inside the photonic crystal. Thus the intensity of photoluminescence can be increased dramatically. The photoluminescence enhancement was also observed for the third arrangement, the mechanism is the same as the second arrangement. The photo-luminescence of $\mathrm{CdSe}$ was passed through the glass slide and was reflected by the silica photonic crystal.

When the angle of photoluminescence collection was varied from $90^{\circ}$ to $45^{\circ}$, the stop band of the photonic crystal was moved to lower wavelengths. Using the second arrangement, we carefully controlled the CdSe nano-particles inside the voids of silica photonic crystal that has the stop band at $520 \mathrm{~nm}$. We have observed that the photoluminescence intensity of $45^{\circ}$ collection is two times larger than that of $90^{\circ}$ collection as shown in Fig. 4. At the $90^{\circ}$ collection, the stop band of silica photonic crystal at $520 \mathrm{~nm}$ inhibited the photoluminescence peak of CdSe at $517 \mathrm{~nm}$. When the collection angle varied to $45^{\circ}$, the stop band of photonic crystal was blue shifted and the photoluminescence of CdSe nanoparticles emitted outside from the stop band of photonic crystal. In this way, one can control the intensity of photoluminescence by changing the angle of the photonic crystal. With a complete photonic crystal band gap and broad enough to overlap with the whole photoluminescence band of nanoparticles, the total inhibition of CdSe photoluminescence will be possible.

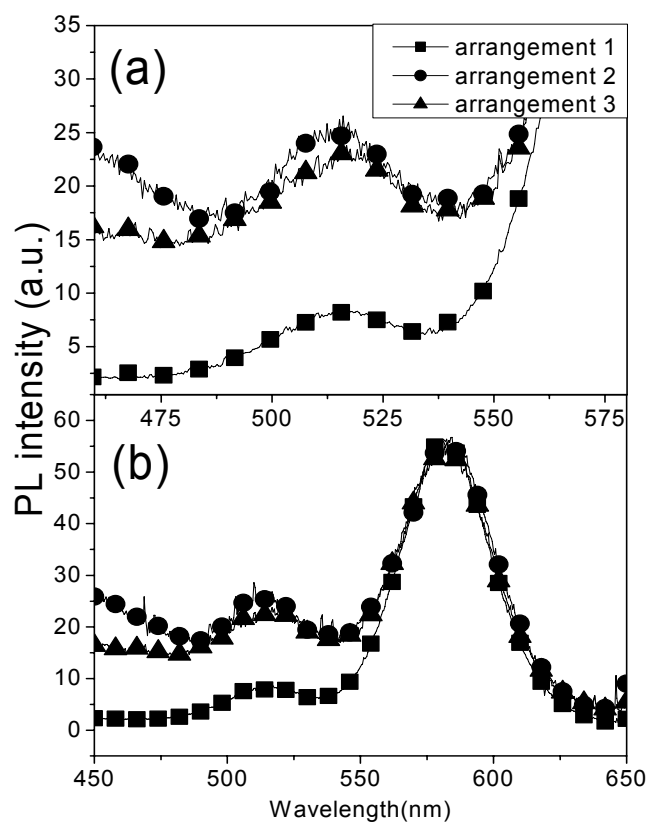

Fig. 3. Photoluminescence spectra of various arrangements of the CdSe nanoparticles respect to photonic crystal with stop band at $490 \mathrm{~nm}$. (a) The photoluminescence intensity at $517 \mathrm{~nm}$ from the arrangement 2 and the arrangement 3 were greatly enhanced by 4 to 5 time as compared to that of arrangement 1 . (b) The full spectra including the standard peak.

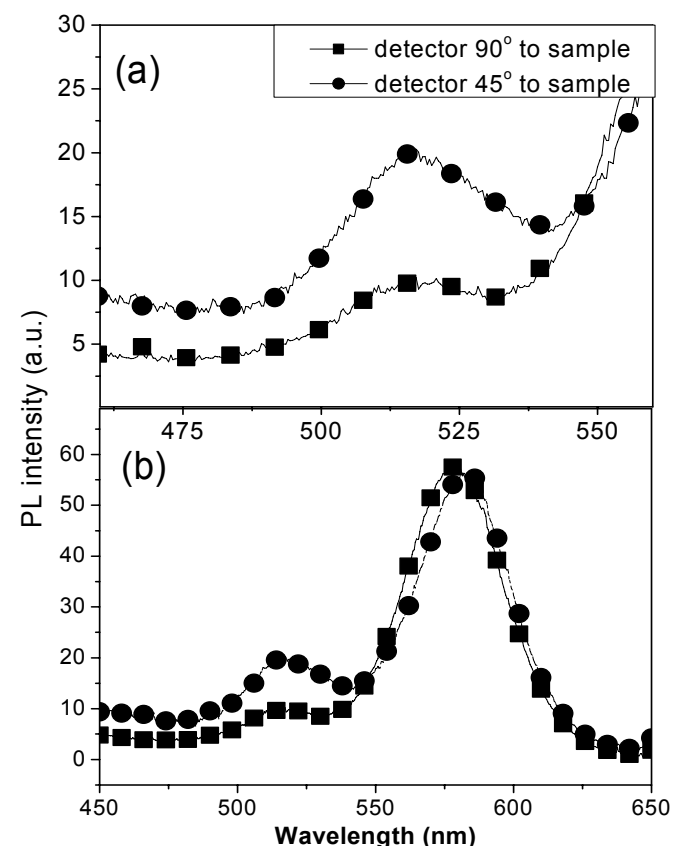

Fig. 4. Photoluminescence spectra were measured at $45^{\circ}$ and $90^{\circ}$ respectively to the $\mathrm{CdSe}$ infiltrated photonic crystal with stop band at $520 \mathrm{~nm}$. (a) The photoluminescence intensity of $517 \mathrm{~nm}$ collected at angle $45^{\circ}$ is larger than that collected at angle $90^{\circ}$. (b) The full spectra including the standard peak. 


\section{CONCLUSION}

In summary, we have demonstrated that the luminescence properties of the CdSe nanoparticles can be controlled by self-assembled 3-D silica photonic crystal. When the emitting wavelength of nanoparticles matches the stop band of photonic crystal, the photoluminescence of the CdSe nanoparticles can be greatly enhanced by up to 5 times. By changing the collection angle of photoluminescence measurements, the photoluminescence intensity of CdSe embedded in photonic crystal can also be controlled.

\section{ACKNOWLEDGEMENT}

We would like to thank National Science Council of Republic of China for financial support of this research under the grants of (NSC93-2102M-002-011)

\section{REFERENCES}

[1] E. Yablonovitch, "Inhibited spontaneous emission in solidstate physics and electronics" Phys. Rev. Lett. vol. 58, pp. 2059, 1987

[2] S. John, "Exact critical behavior of a random bond twodimensional Ising model" Phys. Rev. Lett. vol. 58, pp. 2468, 1987

[3] E. Yablonovitch and T. J. Gmitter, "Virtual photoconductivity due to intense optical radiation transmitted through a semiconductor" Phys. Rev. B, vol. 48, pp. 11265,1993

[4] S. Noda, "Two- and Three-Dimensional Photonic Crystals in III-V Semiconductors" MRS Bull. vol. 26, pp. 618, 2001

[5] A. J. Turberfield, "Photonic Crystals Made by Holographic Lithography" MRS Bull. vol. 26, pp. 632, 2001
[6] Y. A. Vlasov, M. Deutsch, and D.J. Norris, "Single-domain spectroscopy of self-assembled photonic crystals" Appl. Phys. Lett. vol. 76, pp. 1627, 2000

[7] S. H. Park, Y. N. and Xia, "Assembly of Mesoscale Particles over Large Areas and Its Application in Fabricating Tunable Optical Filters" Langmuir vol. 15, pp. 266, 1999

[8] A. Blanco, C. Lopez, R. Mayoral, H. Mÿguez, and F. Meseguer, "Red-light-emitting injection laser based on InP/GaInP self-assembled quantum dots" Appl. Phys. Lett. vol. 73, no. 13, pp. 1784, 1998

[9] M. Miller, R. Zentel, T. Maka, S. G. Romanov, Clivia M., and Sotomayor Torres, "Photonic Crystal Films with High Refractive Index Contrast" Adv. Mat. vol. 12, no. 20, pp. 1499,2000

[10] J. C. Knight, T. A. Birks, P. St. J. Russell and D. M. Atkin, "All-silica single-mode optical fiber with photonic crystal cladding“ Opt. Lett. vol. 21, pp. 1547, 1996

[11] F. Benabid, J. C. Knight, G. Antonopoulos, and P. St. J. Russell, "Stimulated Raman Scattering in Hydrogen-Filled Hollow-Core Photonic Crystal Fiber" Science vol. 298, pp. 399,2002

[12] R. Colombelli*, K. Srinivasan, M. Troccoli, O. Painter, C. F. Gmachl, D. M. Tennant,A. M. Sergent, D. L. Sivco, A. Y. Cho, and F. Capasso, "Quantum Cascade Surface-Emitting Photonic Crystal Laser" Science vol. 302, pp. 1374, 2003

[13] B. Song, S. Noda,* and T. Asano, "Photonic Devices Based on In-Plane Hetero Photonic Crystals" Science vol. 300, pp. 1537,2003

[14] Z. A. Peng and X. Peng, "Formation of High-Quality CdTe, CdSe, and CdS Nanocrystals Using $\mathrm{CdO}$ as Precursor" $J$ Am. Chem. Soc. vol. 123, pp. 183, 2001

[15] W. Stöber, A. Fink, and E. J. Bohn, J. Colloid Interface Sci. vol. 26, pp. 62, 1968.

[16] Y. Xia, B. Gates, and S. H. Park, "Fabrication of threedimensional photonic crystals for use in the spectral region from ultraviolet to near-infrared" $J$. of Lightwave Technology vol. 17, no. 11, pp. 1956, 1999 